\title{
Crystallite size dependent carrier recombination rate and thermal diffusivity in undoped and boron doped CVD diamond layers
}

\author{
P. Ščajev ${ }^{*, 1}$, S. Nargelas' ${ }^{1}$, K. Jarašiūnas ${ }^{1}$, I. Kisialiou² ${ }^{2}$ E. Ivakin², W. Deferme ${ }^{3,4}$, J. D’Haen ${ }^{3,5}$, and K. Haenen ${ }^{3,5}$ \\ ${ }^{1}$ Institute of Applied Research, Vilnius University, 10222 Vilnius, Lithuania \\ ${ }^{2}$ Institute of Physics, Academy of Sciences of Belarus, 220072 Minsk, Belarus \\ ${ }^{3}$ Institute for Materials Research (IMO), Hasselt University, 3590 Diepenbeek, Belgium \\ ${ }^{4}$ XIOS University College Limburg, 3590 Diepenbeek, Belgium \\ ${ }^{5}$ IMOMEC, IMEC vzw, 3590 Diepenbeek, Belgium
}

Received 1 April 2013, revised 30 April 2013, accepted 31 May 2013

Published online 5 August 2013

Keywords carrier diffusion, diamond, differential transmittivity, light-induced transient gratings, recombination, reflectivity

*Corresponding author: e-mail patrik.scajev@ff.vu.lt, Phone: +370 5 2366036, Fax: +370 52366037

Carrier dynamics under interband carrier injection conditions $(213 \mathrm{~nm})$ was studied in undoped and boron-doped microcrystalline diamond layers with different grain size. The grain size, determined by scanning electron microscopy and electron backscattered diffraction, varied from $130 \mu \mathrm{m}$ on the growth side to $\sim 1-2 \mu \mathrm{m}$ on the nucleation side of a $1.0 \mathrm{~mm}$-thick undoped layer. Carrier lifetimes measured by differential transmittivity (DT) technique varied from 1 to $4 \mathrm{~ns}$ on the growth side to $\sim 220 \mathrm{ps}$ on the nucleation side. Also the carrier diffusivity was found higher on the growth side. The B-doped layer with $40 \mu \mathrm{m}$ grain size at the growth side exhibited 380 ps carrier lifetime, which decreased to $130 \mathrm{ps}$ on the nucleation side. Even shorter lifetimes $(\sim 100-200 \mathrm{ps}$ and $\sim 10 \mathrm{ps}$, correspondingly) were revealed in this layer by differential reflectivity decay due to impact of subsurface defects. Therefore we conclude that the recombination rate in presence of large grains is dominated by bulk non-radiative traps, as diffusion time of carriers to reach grain boundaries is much longer (few $\mu s$ ). An impact of grain boundaries to recombination is expected in case of smaller grains. Thermal grating decay provided values of thermal diffusivity $D_{\text {th }}$ in the range of $12-6 \mathrm{~cm}^{2} \mathrm{~s}^{-1}$, respectively, on the growth and nucleation side of the layers, and its decrease with reduction of grain size was attributed to phonon scattering on grain boundaries.
1 Introduction Carrier and thermal dynamics in diamonds are usually investigated at high photoexcitation conditions using above the bandgap quantum energies $\left(h v>E_{\mathrm{g}}=5.5 \mathrm{eV}\right)$ for carrier injection. The determined carrier lifetimes of 0.14-3.3 ns in HPHT and CVD grown diamonds $[1,2]$ revealed a correlation between the carrier lifetimes and the nitrogen density, suggesting that nitrogenrelated defects act as main carrier recombination centers in diamond $[3,4]$.

In order to study carrier lifetimes and diffusivity at lower injected carrier densities, two-photon carrier injection (2P) by a picosecond laser pulse at $351 \mathrm{~nm}$ was recently applied [5]. In this way, excitation of the entire bulk crystal was achieved [6]. In the undoped microcrystalline (U-CVD) sample, the fast (80 ps) and slow (3-8 ns) recombination transients were observed and attributed to bulk intra-grain defects. A presence of the fast component was explained by non-uniform defect distribution along the sample thickness.

In present work, we use interband injection at $213 \mathrm{~nm}$ to inject carriers into few- $\mu \mathrm{m}$ depth of a U-CVD crystal and thus discriminate recombination rates on the growth and the nucleation side, typically having grains of different size. Lifetime correlation with the grain size was determined by monitoring carrier dynamics at both surfaces. Additionally we performed studies on a heavily boron doped microcrystalline layer. Lifetime was found to be dominated by point defects in the grains. Impact of surface defects was measured by differential reflectivity and provided much faster recombination rates.

Thermal and carrier diffusivities were investigated on both sides of the samples by using the light-induced transient grating technique. Injection dependence of carrier diffusivity 
on the less-defective growth side and nucleation sides of undoped sample revealed dominance of many body effects [7] and an impact of grain boundaries, respectively. The thermal diffusivity, $D_{\text {th }}$, was lower in case of smaller crystallites due to scattering on grain boundaries.

2 Samples and experimental techniques Carrier dynamics under interband carrier injection conditions $(213 \mathrm{~nm})$ was studied in two freestanding microcrystalline layers: an undoped, $1.0-\mathrm{mm}$ thick (110) textured U-CVD diamond $[8,9]$ and in boron doped $0.25-\mathrm{mm}$ thick B-CVD diamond with $4-9 \times 10^{18} \mathrm{~cm}^{-3} \mathrm{~B}$ concentration. The growth sides of both samples were polished. Full details in the deposition conditions of the samples can be found in Ref. [9]. The average grain size, determined by scanning electron microscopy (SEM), a FEI Quanta 200 FEG-SEM with HKL EBSD system with Nordlys camera (Fig. 1), varied from $d_{\text {grain }} \sim 1-2 \mu \mathrm{m}$ to $\sim 130 \mu \mathrm{m}$ on the nucleation and growth sides of the undoped layer, while in the B-doped layer the grain size at the growth side was $\sim 40 \mu \mathrm{m}$ (the average grain area, $\sim \pi d_{\text {grain }} / 4$, was obtained dividing the total investigated area by number of grains in it).

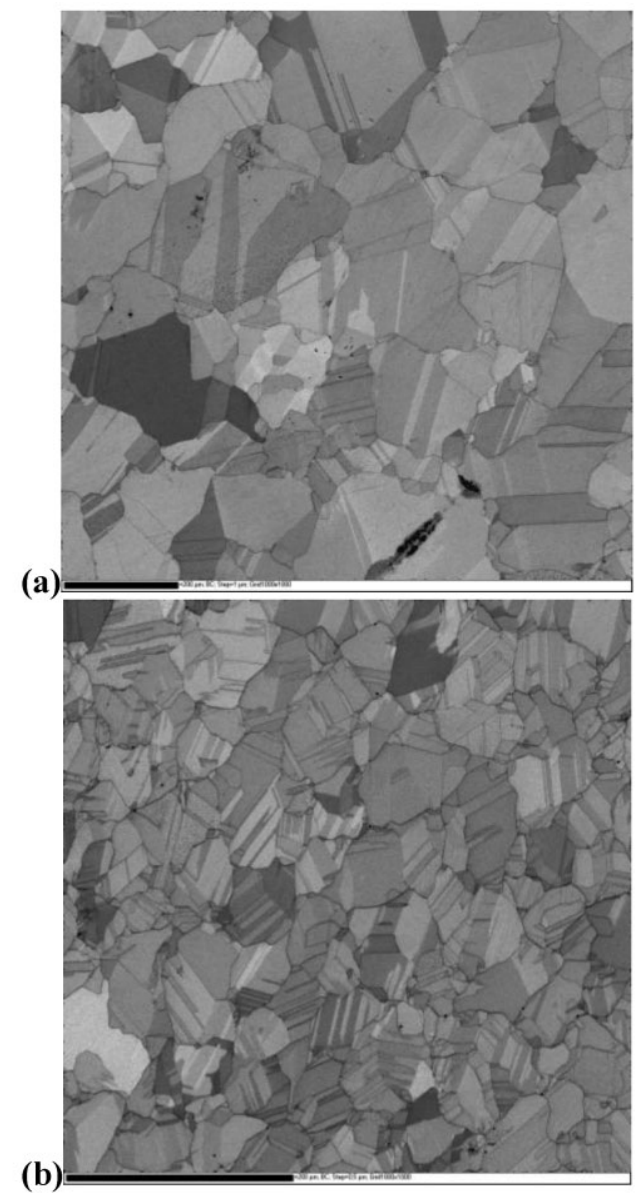

Figure 1 Microcrystalline structures on the growth side of the undoped (a) and B-doped CVD layers (b). The bars in the figures indicate a $200 \mu \mathrm{m}$ length.
For carrier injection, a Nd:YAG laser (operating at $\lambda_{1}=1064 \mathrm{~nm}$ with $10 \mathrm{~Hz}$ repetition rate) was used. Its 5-th harmonic pulse $\lambda_{5}=213 \mathrm{~nm}\left(\tau_{1 \mathrm{~h}}=25 \mathrm{ps}\right)$ generated the carriers in the bulk via interband transitions, providing their density in $5 \times 10^{17}$ to $3 \times 10^{19} \mathrm{~cm}^{-3}$ range. Interband carrier generation at $\lambda_{5}$ in a $\delta=1 / \alpha_{5}=3.1 \mu \mathrm{m}$ thick surface region (here $\alpha_{5}=3200 \mathrm{~cm}^{-1}$ is the absorption coefficient for $213 \mathrm{~nm}$ in undoped sample, determined using diffraction based technique [10]), provided free carriers, and their lifetimes and diffusivities were measured on the both sides of the studied samples. The optically delayed (up to $4 \mathrm{~ns}$ ) picosecond probe pulse at $\lambda_{1}=1064 \mathrm{~nm}$ or $\lambda_{2}=532 \mathrm{~nm}$ wavelength was used to monitor the fast free carrier decay transients.

Light-induced free carrier transient gratings with varying grating period $\Lambda$ were recorded by light interference pattern [11]. In this way, spatially-modulated carrier density $N(x, z)=\Delta N_{0}[1+\cos (2 \pi x / \Lambda)] \times \exp \left(-\alpha_{5} z\right)$ was generated by interband transitions [6]. Here $\Delta N_{0}=\alpha_{5} I_{0} / h v_{5}$ is the carrier density near surface $(z=0)$, where $h v_{5}=5.82 \mathrm{eV}$ is the quantum energy of $213 \mathrm{~nm}$ wavelength, $I_{0}$ is the excitation fluence. Carrier density averaged over the excited depth shortly after excitation was equal to $\Delta N_{\mathrm{av}}=\Delta N_{0} / 2$. Probe beam diffraction efficiency on the free-carrier grating $\eta \sim \Delta N_{0}^{2} \exp \left(-2 t / \tau_{\mathrm{GC}}\right)$ provided the grating decay time $\tau_{\mathrm{GC}}$ at different grating periods $\Lambda$, enabling determination of carrier diffusion coefficient $D_{\mathrm{a}}$ and carrier lifetime $\tau_{\mathrm{R}}$ according to a well known relationship $1 / \tau_{\mathrm{GC}}=1 / \tau_{\mathrm{R}}+4 \pi^{2} D_{\mathrm{a}} / \Lambda^{2}[11]$.

For thermal grating recording nearby the surface, the $213 \mathrm{~nm}$ wavelength of a picosecond $\mathrm{Nd}$ :YAG laser $\left(\tau_{1 \mathrm{~h}}=60 \mathrm{ps}\right)$ was used, and the grating decay was monitored by $\mathrm{CW}$ InGaN laser at $473 \mathrm{~nm}$. For comparison with the thermal grating decay in the bulk, excitation at $266 \mathrm{~nm}$ was used. The diffusive decay of the transient thermal grating (TTG), $1 / \tau_{\mathrm{GT}}=4 \pi^{2} D_{\mathrm{th}} / \Lambda^{2}$, provided the thermal diffusivity $D_{\text {th }}$ [12]. TTG decay was also measured in a reflection mode (TTG-R) $[13,14]$.

The differential transmittivity (DT) kinetics were used to determine carrier lifetime values $\tau_{\mathrm{R}}$ at various temperatures and excitations. In the given case, carriers were injected by a Gaussian beam and DT decay was given by $\ln \left(T_{0} / T(t)\right)=\sigma_{\mathrm{eh}} \delta N_{0} \exp \left(-t / \tau_{\mathrm{R}}\right)$ relation, where $\sigma_{\mathrm{eh}}=$ $\sigma_{\mathrm{e}}+\sigma_{\mathrm{h}}=8.8 \times 10^{-18} \mathrm{~cm}^{2}$ is the total free carrier absorption (FCA) cross section [6], composed of electron $\left(\sigma_{\mathrm{e}}\right)$ and hole $\left(\sigma_{\mathrm{h}}\right)$ absorption cross sections. To elucidate impact of surface traps, differential reflectivity (DR) measurements were performed [15]. In the latter case, the near-surface carrier density $(\Delta N(z=0, t))$ was probed, as the DR signal is described by a relation $\Delta R / R \sim \Delta N(z=0, t)$ (here $R$ is the reflectivity coefficient).

\section{Results and discussion}

3.1 DT and DR decays and lifetimes Differential transmittivity and reflectivity signals exhibited linear injection dependencies thus the signal decay time precisely reflected carrier lifetime. Decay kinetics for the undoped 
CVD sample are shown in Fig. 2a and b. Decay time on the growth side exhibited very strong position dependence, while such dependence on the nucleation side was much weaker. This effect is probably due to more pronounced variation of grain size on the growth side, as can be seen in Fig. 1a. Lifetime dependence on injection, if measured in the

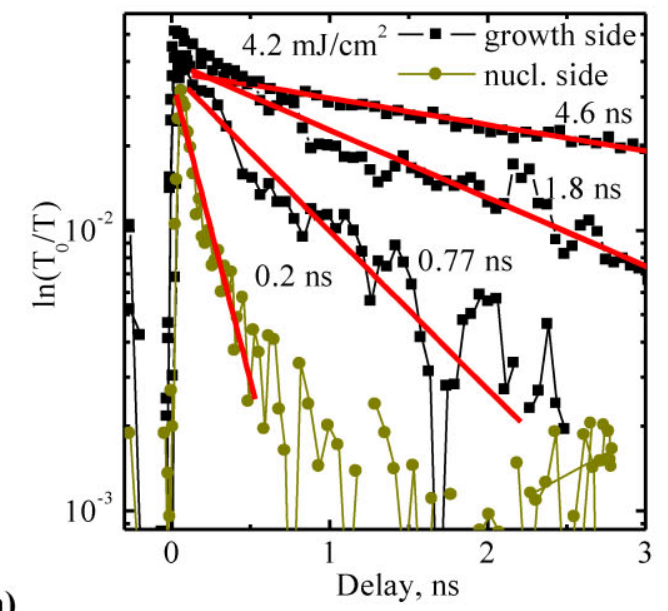

(a)

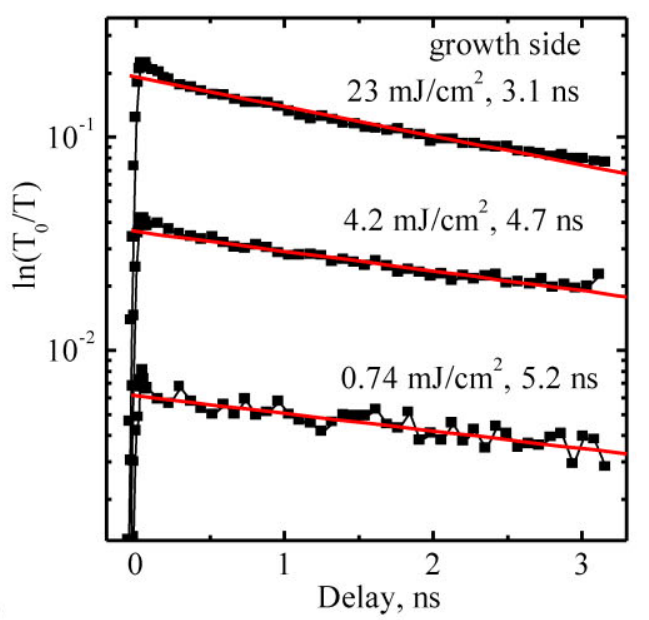

(b)
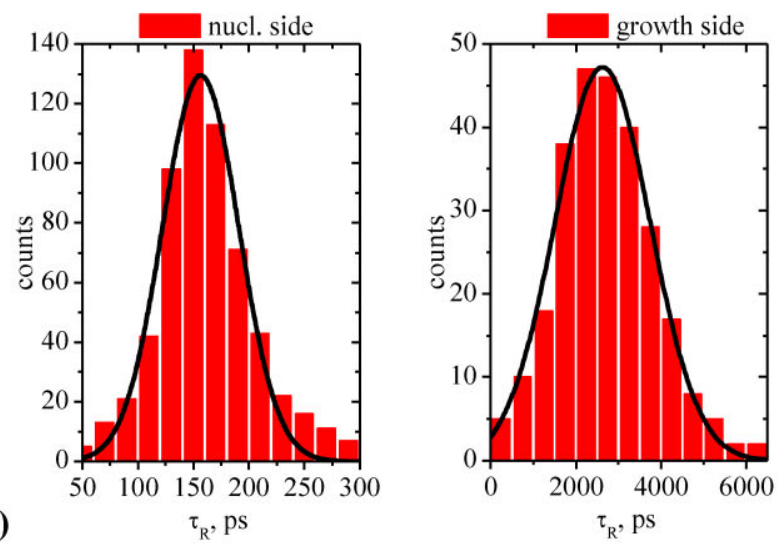

Figure 2 Carrier lifetimes on the growth and the nucleation sides of the undoped layer, measured at $I_{0}=10 \mathrm{~mJ} \mathrm{~cm}^{-2}$ excitation fluence (a), DT kinetics at different $I_{0}$ values (b), and statistics of lifetime variation on the opposite sides of the layer. selected place with the longest lifetime, was rather weak (Fig. 2b), thus the decay of 3-5 ns is determined by nonradiative recombination, consisting of recombination on structural, point defects, and surface. Initial slightly faster decay can be a consequence of some different grain sizes (and consequently, lifetimes) as well of surface defects. We note that the impact of surface defects leads to the decreased lifetimes, as revealed by DR kinetics.

To evaluate the statistical distribution of lifetime and its correlation with grain size, the lifetimes were measured in 280 points, using a probe of $\sim 25 \mu \mathrm{m}$ diameter. For this purpose in each point the DT values were measured at two different delay times ( 0.5 and $2.5 \mathrm{~ns}$ for the growth side and 0.1 and $0.4 \mathrm{~ns}$ for the nucleation side, respectively), thus providing a simple way for carrier lifetime determination. We determined an almost Gaussian lifetime distribution on the nucleation and growth sides, with the average lifetime values, $\tau_{\mathrm{av}}$, of 160 and $2600 \mathrm{ps}$, respectively (the lifetime fitting as $A \times \exp \left[-2 \times\left(\left(\tau-\tau_{\mathrm{av}}\right) / \sigma\right)^{2}\right]$ was used, here $\sigma=70 \mathrm{ps}$ and $2200 \mathrm{ps}$, respectively). Consequently, the average lifetime on the nucleation side is $\sim 15$ times smaller than that on the growth side, while the grain size on the nucleation side is about $\sim 100$ times smaller (few $\mu \mathrm{m}$ with respect to few hundred $\mu \mathrm{m}$ on the growth side). We conclude that in the case of large grains the recombination is governed by bulk non-radiative traps [16], as the carrier diffusion time to grain boundaries $\left(\tau_{\mathrm{D}}=d_{\text {grain }}{ }^{2} /\left(22 \times D_{\mathrm{a}}\right)[17]\right)$ is much larger $(\sim 0.1 \mu \mathrm{s}$ and $\sim 1 \mu$ s, respectively), while on the nucleation side an impact of diffusion-limited recombination on grain boundaries is possible, as $\tau_{\mathrm{D}}=100-400 \mathrm{ps}$ [17]. Indeed, lifetimes up to $100 \mathrm{~ns}$ were observed in $\sim 100 \mu \mathrm{m}$ large grains of purified CVD diamond at $266 \mathrm{~nm}$ excitation wavelength [18]. For comparison, in bulk HPHT diamonds lifetimes were in 150$330 \mathrm{~ns}$ range [5]. The large variation of lifetime values (especially on the growth side) might be a consequence of different growth orientation of the grains and consequently different point defect incorporation probability [19].

In the B-CVD sample, the lifetime values exhibited a rather narrow distribution on both sides. Lifetime value on the nucleation side was 3 times smaller partially due to smaller grains there when compared to the growth side. However, by order of magnitude shorter lifetime on growth side of the B-CVD in comparison to the U-CVD indicates for much higher defect density in the B-CVD (i.e. dislocations, other diamond phases), which is caused by high boron concentration [20]. For DT measurements (Fig. 3a) in this sample we used a $532 \mathrm{~nm}$ probe, as the layer transmission for the $1064 \mathrm{~nm}$ probe was not detectable due to a relatively high boron concentration. DR measurements (Fig. 3b) provided $97 \mathrm{ps}$ on the growth and $<10 \mathrm{ps}$ on the nucleation side. We note that DR decay in the undoped layer provided lifetimes of $250 \mathrm{ps}$ on the growth and $<10 \mathrm{ps}$ on the nucleation sides. The growth surface lifetimes are comparable for both samples, but are much shorter than the bulk lifetime, probably due to similar but large surface trap densities. 


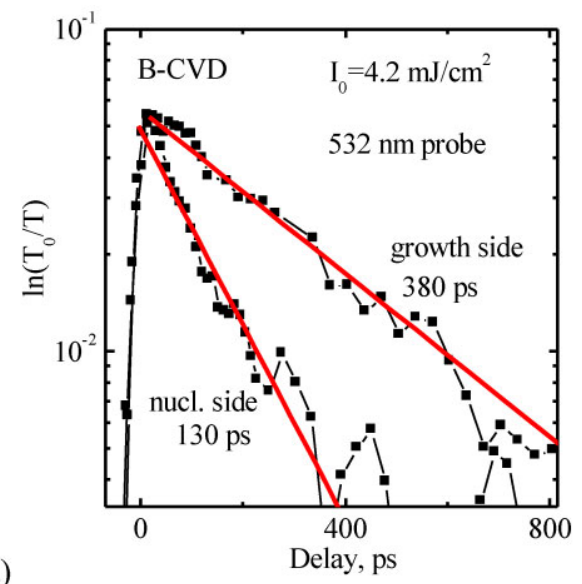

(a)

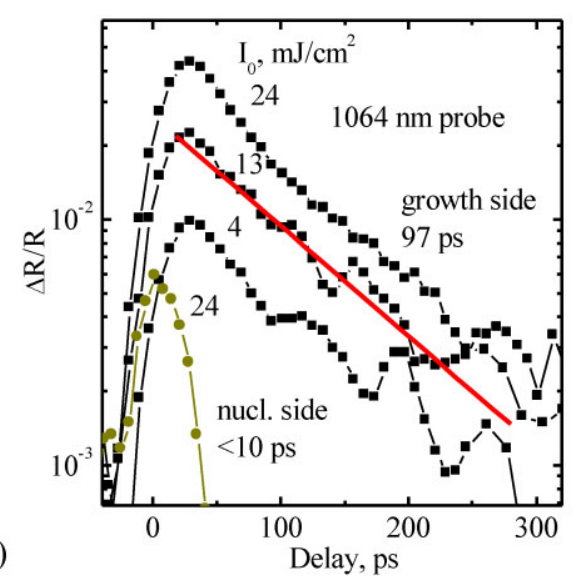

Figure 3 Differential transmittivity (a) and reflectivity (b) decay on the growth and nucleation sides of B-doped CVD layer.

3.2 Carrier diffusivity measurements TG decay time $\tau_{\mathrm{GC}}$ was measured for two grating periods (equal to $\Lambda=1.72$ and $6.9 \mu \mathrm{m}$ ) and provided $\tau_{\mathrm{GC}}$ values in the $0.1-$ $2 \mathrm{~ns}$ range. The grating decay was diffusion-governed on the growth side and recombination governed on the nucleation side of the undoped layer (the optical quality of the B-CVD sample was too low to separate the diffracted probe beam from the scattered light background). Dependence of $D_{\mathrm{a}}$ on injection level in the U-CVD is shown on Fig. 4. In turn, the ambipolar mobility can be calculated using the Einstein relationship, $D_{\mathrm{a}}=\mu_{\mathrm{a}} k_{\mathrm{B}} T / e$ [21].

We observed injection-dependent diffusivity: its gradual decrease up to $10^{19} \mathrm{~cm}^{-3}$ of excess carrier density and subsequent increase (Fig. 4). A mobility decrease is due to bandgap renormalization and electron-hole scattering while its increase is due to electron-hole plasma degeneracy and screening of electron-hole scattering (the theoretical curve is taken from our previous work [7]). Hence, the impact of structural and point defects is practically insignificant on the growth side. In contrary, the diffusivity on the nucleation side was rather low and difficult to extract due to short carrier lifetimes $(\sim 100 \mathrm{ps})$. The low value can be explained by

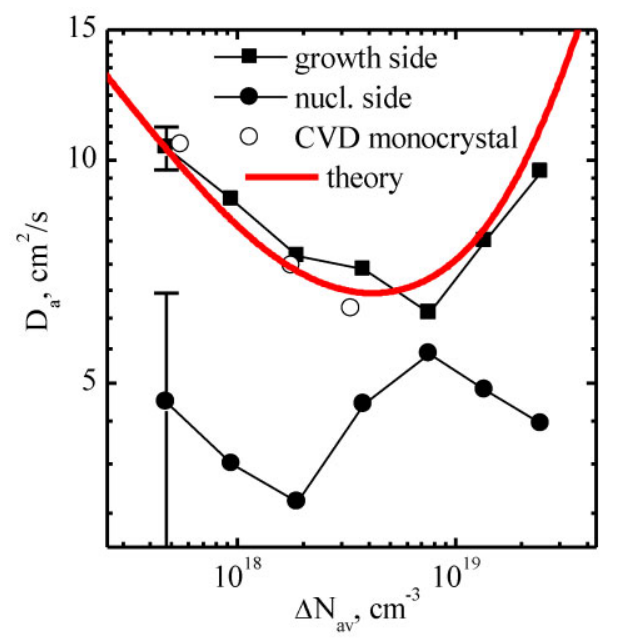

Figure 4 Injection dependencies of ambipolar diffusivity in the U-CVD sample on the growth and nucleation side with large and small crystallite sizes, respectively. Open points are data for undoped monocrystalline CVD diamond [7]. The solid curve was theoretically calculated for undoped diamond [7].

additional scattering of carriers on grain boundaries [22] and more abundant defect density.

3.3 Thermal diffusivity measurements In previous experiments, in-plane thermal diffusivity $D_{\text {th }}$ has been measured by TTG technique in natural diamonds with high defect density [23] and in isotopically enriched HPHT-crystals [24], using for excitation 9.2 and $4.1 \mu \mathrm{m}$ wavelengths, respectively, which were favourable for heat generation. In this work, thermal gratings with $\Lambda=47.5 \mu \mathrm{m}$ period were recorded and diffracted signal was easily detected in the undoped and B-doped layers at $213 \mathrm{~nm}$ and excitation density $I_{0}=10 \mathrm{~mJ} \mathrm{~cm}^{-2}$. Kinetics of homodyne-amplified diffracted beam [12] are shown in Fig. 5. The fast free carrier recombination (see Figs. 2a and 3 a) provided heat which created spatial modulation of the refractive index. In the U-CVD sample, diffusivity was found to be two times larger on the growth side (Fig. 5a) due to larger crystallites. Recording of thermal grating with $\Lambda=50 \mu \mathrm{m}$ in the same layer but at $266 \mathrm{~nm}$ excitation provided the $D_{\mathrm{th}}=9.0 \pm 0.5 \mathrm{~cm}^{2} \mathrm{~s}^{-1}$ value, being average over the sample depth. Therefore, the latter value was the same if measured on growth or nucleation side and close to an average of the surface diffusivities, indicating that the crystallite size continuously increases with thickness, as known from the Van der Drift growth mechanism [25]. Thermal gratings in reflection mode provided very similar $D_{\text {th }}$ values $\left(12.5\right.$ ad $\left.7.3 \mathrm{~cm}^{2} \mathrm{~s}^{-1}\right)$ within the error margins.

As stated before, the optical absorption due to the B-doping prevents measurements in transmission mode. So, we were not able to measure thermal grating decay in transmission mode; instead, we measured TTG decay in reflection mode (Fig. 5b). The decay kinetics are decorated 


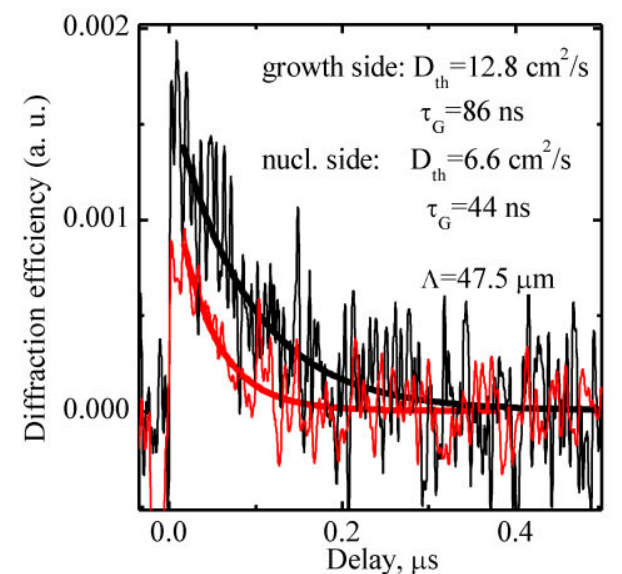

(a)

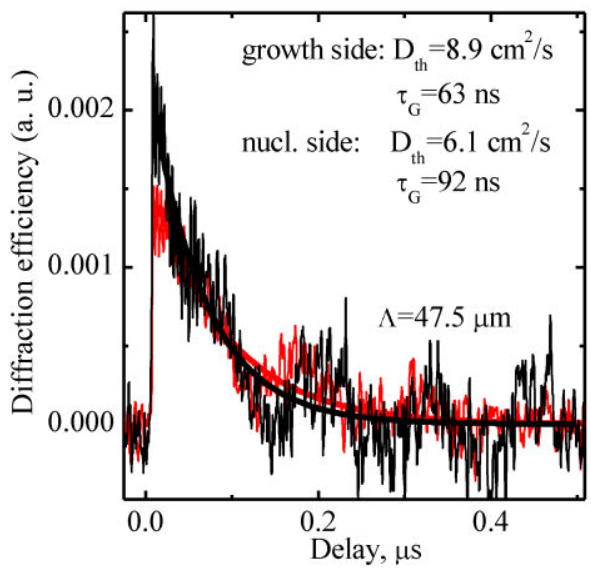

(b)

(c)

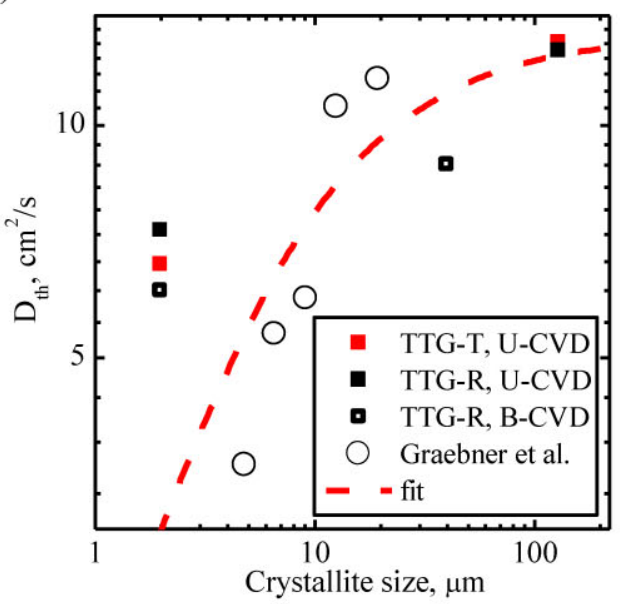

Figure 5 Diffraction kinetics on transient gratings in undoped and B-doped samples in transmission (a) and reflection (b) geometry, respectively. In (c), thermal diffusivity variation with grain size is shown.

by oscillations due to heating of the air at the sample surface (750 MHz, $350 \mathrm{~m} \mathrm{~s}^{-1}$ ) and due to surface acoustic waves (227 MHz, $10.8 \mathrm{~km} \mathrm{~s}^{-1}$ [26]).

Finally we plotted the $D_{\text {th }}$ dependence on crystallite size in the studied samples and compared it with data from
Ref. [27]. At large grain size (e.g. on the growth side of U-CVD), the $D_{\text {th }}$ value was limited by lattice properties; while for smaller grains phonon scattering on grain boundaries prevailed. The presence of other defects, e.g. boron scattering in B-CVD sample, can also have an impact [28]. The fitting curve was obtained composing these two effects, $D_{\text {th }}=1 /(1 / 13+0.5 /$ grainsize $[\mu \mathrm{m}]) \mathrm{cm}^{2} \mathrm{~s}^{-1}$. The obtained $D_{\text {th }}$ values for large crystallites are close to that for a natural single crystalline diamond $\left(D_{\mathrm{th}}=12.4 \mathrm{~cm}^{2} \mathrm{~s}^{-1}[29]\right)$.

4 Conclusions We investigated peculiarities of free carrier recombination, carrier and thermal diffusion processes in undoped and boron doped microcrystalline CVD diamond films at interband excitation. Non-radiative recombination in the samples was attributed to bulk traps within the grains (which are more abundant in small ones), while surface lifetime was limited by surface defects. Carrier diffusivity in the undoped layer with large crystallites was governed by phonon and many body effects while additional scattering on grain boundaries was observed on the nucleation side with few-micrometer size crystallites. The thermal diffusivity also revealed the impact of grain boundary scattering on sample sides with small grains, while few hundred times larger crystallites provided the thermal diffusivity of $\sim 13 \mathrm{~cm}^{2} \mathrm{~s}^{-1}$, being typical for a bulk diamond. Therefore, the growth side of the undoped microcrystalline layer exhibited properties of a bulk diamond as its carrier and thermal diffusivities were limited only by intrinsic properties, although the carrier lifetime was relatively short due to impact of intra-grain defect centers.

Acknowledgements We acknowledge support of Lithuanian Science Council under Grant No. TAP LLT 05/2012. The sample growth was funded by the Research Foundation-Flanders (G.0555.10N) and the EU FP7 through the Marie Curie ITN "MATCON" (PITN-GA-2009-238201), and the Collaborative Project "DIAMANT" (No. 270197).

\section{References}

[1] T. Malinauskas, K. Jarašiūnas, E. Ivakin, N. Tranchant, and M. Nesladek, Phys. Status Solidi A 207, 2058 (2010).

[2] T. Malinauskas, K. Jarašiūnas, E. Ivakin, V. Ralchenko, A. Gontar, and S. Ivakhnenko, Diam. Relat. Mater. 17, 1212 (2008).

[3] L. S. Pan, D. R. Kania, P. Pianetta, J. W. Ager, III M. I. Landstrass, and S. Han, J. Appl. Phys. 73, 2888 (1993).

[4] T. Teraji, S. Yoshizaki, S. Mitani, T. Watanabe, and T. Ito, J. Appl. Phys. 96, 7300 (2004).

[5] P. Ščajev, V. Gudelis, K. Jarašiūnas, I. Kisialiou, E. Ivakin, M. Nesladek, and K. Haenen, Phys. Status Solidi A 209, 1744 (2012).

[6] P. Ščajev, V. Gudelis, E. Ivakin, and K. Jarašiūnas, Phys. Status Solidi A 208, 2067 (2011).

[7] P. Ščajev, T. Malinauskas, L. Lubys, E. Ivakin, M. Nesladek, K. Haenen, and K. Jarašiū nas, Phys. Status Solidi RRL 5, 193 (2011). 
[8] N. Habka, J. Barjon, A. Lazea, and K. Haenen, J. Appl. Phys. 107, 103531 (2010).

[9] K. Haenen, A. Lazea, J. Barjon, J. D’Haen, N. Habka, T. Teraji, S. Koizumi, and V. Mortet, J. Phys.: Condens. Matter 21, 364204 (2009).

[10] P. Ščajev, M. Kato, and K. Jarašiūnas, J. Phys. D: Appl. Phys. 44, 365402 (2011).

[11] C. A. Hoffman, K. Jarašiūnas, H. J. Gerritsen, and A. Nurmikko, Appl. Phys. Lett. 33, 536 (1978).

[12] E. V. Ivakin, A. V. Sukhodolov, V. G. Ralchenko, A. V. Vlasov, and A. V. Khomich, Quantum Electron. 32, 367 (2002).

[13] Q. Shen, A. Harata, and T. Sawada, J. Appl. Phys. 77, 1488 (1995).

[14] O. W. Käding, H. Skurk, A. A. Maznev, and E. Matthias, Appl. Phys. A 61, 253 (1995).

[15] A. J. Sabbah and D. M. Riffe, Phys. Rev. B 66, 165217 (2002).

[16] M. Bruzzi, F. Hartjes, S. Lagomarsino, D. Menichelli, S. Mersi, S. Miglio, M. Scaringella, and S. Sciortino, Phys. Status Solidi A 199, 138 (2003).

[17] P. Ščajev, A. Usikov, V. Soukhoveev, R. Aleksiejūnas, and K. Jarašiūnas, Appl. Phys. Lett. 98, 202105 (2011).

[18] J. Hammersberg, J. Isberg, E. Johansson, T. Lundström, O. Hjortstam, and H. Bernhoff, Diam. Relat. Mater. 10, 574 (2001).
[19] A. M. Zaitsev, Optical Properties of Diamond: A Data Handbook (Springer, Berlin, 2001).

[20] W. L. Wang, M. C. Polo, G. Sánchez, J. Cifre, and J. Esteve, J. Appl. Phys. 80, 1846 (1996).

[21] J. F. Schetzina and J. P. McKelvey, Phys. Rev. B 2, 1869 (1970).

[22] N. G. Weimann and L. F. Eastman, J. Appl. Phys. 83, 3656 (1998).

[23] V. V. Gorbachev, V. M. Durasov, R. B. Zezin, E. V. Ivakin, A. S. Rubanov, and N. A. Tatyanina, Phys. Status Solidi B 150, 901 (1988).

[24] A. Tokmakoff, W. F. Banhplzer, and M. D. Fayer, Appl. Phys. A 56, 87 (1993).

[25] C. Wild, P. Koidl, W. Müller-Sebert, H. Walcher, R. Kohl, N. Herres, R. Locher, R. Samlenski, and R. Brenn, Diam. Relat. Mater. 2, 158 (1993).

[26] V. Mortet, O. A. Williams, and K. Haenen, Phys. Status Solidi A 205, 1009 (2008).

[27] J. E. Graebner, Int. J. Thermophys. 19, 511 (1998).

[28] E. Wörner, E. Pleuler, C. Wild, and P. Koidl, Diam. Relat. Mater. 12, 744 (2003).

[29] T. R. Anthony, W. P. Banholzer, J. F. Fleischer, L. Wei, P. K. Kuo, R. L. Thomas, and R. W. Pryor, Phys. Rev. B 42, 1104 (1990). 